\title{
Allergic eye disease: Blocking LTB4/C5 in vivo suppressed disease and Th2 \& Th9 cells
}

To the Editor

Vernal keratoconjunctivitis (VKC) affects children and can impair vision if the cornea becomes involved. Immunosuppressives (steroids and cyclosporin $\mathrm{A}$ ) are required but can have side effects, and novel immunotherapeutic approaches are needed. ${ }^{1}$ The aim of this study was to investigate the contributions of LTB4 and complement C5 in a model of allergic eye disease (experimental allergy conjunctivitis, EAC). ${ }^{2}$ Previous studies have demonstrated that nomacopan, a bifunctional recombinant biologic derived from blood-feeding ticks, has anti-inflammatory properties by capturing LTB4 and preventing it from interacting with its two known $\mathrm{G}$ protein-coupled cell surface receptors (GPCR BLT1 and BLT2). Simultaneously, nomacopan inhibited $\mathrm{C} 5$ end terminal complement activation, thereby preventing formation of C5b-9 and C5a. ${ }^{3}$ These two pathways have evolutionary connections as phylogenetic analysis showed that tick saliva started as an LTB4 inhibitor, and subsequently acquired the ability to inhibit C5. It has been shown in a mouse model of inflammatory arthritis that C5a production resulted in the release of LTB4 to promote further neutrophil migration to the interstitium. ${ }^{4}$

EAC is a model of allergic eye disease mainly driven by effector Th2 cells and mast cells (MC). During EAC, conjunctival inflammation can be detected after 5 days, scored non-invasively ${ }^{2}$ (Figure S1AC), with elevated levels of conjunctival IL-9-expressing CD4 ${ }^{+} \mathrm{T}$ cells and $M C$ detected in tissues and cells expanded from conjunctival explants (Figure S1D-J). Significantly increased levels of tryptase ${ }^{+}$ conjunctival MC were observed, co-expressing intracellular IL-9 in the sub-epithelial area within the fornix of conjunctival EAC tissues (Figure S1G).

In this study, we investigated the effects of topically administered nomacopan in EAC and observed a significant suppression of disease in treated mice, and a decrease in IL-9-expressing $\mathrm{CD} 4^{\mathrm{T}} \mathrm{T}$ cells (Figure $1 \mathrm{~A}-\mathrm{C}$; Figure S2A). Recent studies have demonstrated that IL-9 can be produced by Th9 and Th2 cells. ${ }^{5,6}$ To determine which $\mathrm{CD} 4^{+} \mathrm{T}$ cell subset was producing IL-9, transcription factor expression was investigated. IL-9-producing Th2 cells express GATA3, but not PU-1, whereas IL-9-producing Th9 cells express PU.1, but not GATA3. Within the infiltrating CD $4^{+} \mathrm{T}$ cells in EAC, Th9 cells (IL-9 ${ }^{+}$PU. $1^{+}$) and IL-9-expressing Th2 cells $\left(\right.$ GATA $\left.3^{+} \mathrm{IL}-4^{+} \mathrm{IL}^{-} 9^{+}\right)$cells were increased in OVA-challenged mice compared with controls (Figure $\mathrm{S} 1 \mathrm{H}-\mathrm{J}$ ).
During early stages of EAC (Days 1-6), no effect by nomacopan was observed due to low inflammatory scores overall. However, at days 7 and 10, a significant decrease in IL- $9^{+} \mathrm{CD} 4^{+} \mathrm{T}$ cells (Figure 1CF) following nomacopan treatment was observed. The levels of Th2 $\left(\mathrm{IL}^{-} 4^{+}\right.$PU.1- ${ }^{-} \mathrm{GATA3}^{+}$) cells and IL-9-expressing Th2 cells were also significantly reduced (Figure S2B-D). Interestingly, although there was an overall reduction in IL-9 expression levels, there were significant changes in Th9 (IL-9 $9^{+} \mathrm{PU} .1^{+} \mathrm{IL}-4^{-}$) levels only at the higher concentration of nomacopan (Figure 1D).

This in vivo model shares many features with VKC in man since there is a predominant $C D 4^{+} T$ cell infiltration of the conjunctival tissues and evidence of fibrosis. ${ }^{2}$ Hence, we used VKC tissue specimens and tear fluids to compare with EAC tissues. Tears collected from VKC patients during active disease showed a significantly higher level of IL-9 (clinically uncontrolled) as compared with inactive VKC (controlled; Figure 2A). VKC conjunctival tissue sections were examined for expression of CD4, IL-9, IL-9 receptor (IL-9R), and tryptase (Figure 2B-E). IL-9-expressing CD4 ${ }^{+} \mathrm{T}$ cells and $\mathrm{MC}$ were both significantly up-regulated in all active VKC specimens $(n=7)$ as compared with controls. We observed CD4 ${ }^{+} T$ cells co-localized with IL-9 in the sub-epithelial and stromal areas of VKC sections (Figure 2B) and IL9R expression (Figure 2C). Infiltrating $\mathrm{MC}$ (Tryptase ${ }^{+}$) and other cell types also expressed IL-9R (Figure 2D) within stromal areas, with the frequency of IL-9R-expressing MC significantly higher than IL-9Rexpressing $C D 4^{+} \mathrm{T}$ cells (Figure $2 \mathrm{E}$ ).

To further investigate whether the receptors for those ligands targeted by nomacopan were expressed during EAC, conjunctival tissue expression and localization of C5aR and BLT1 (LTB4 receptors) were investigated (Figure S2E) as well as in VKC and healthy tissue controls (Figure 2F,G). Relative expression of BLT1 and C5aR was analysed by counting positively stained cells in at least 5 nonoverlapping areas (ImageJ). Interestingly, increases in infiltrating immune cells within the stromal areas correlated with increased expression of BLT1 (means \pm SD; $34.98 \pm 15)$ and C $5 \mathrm{aR}(56.67 \pm 12)$ in EAC, and comparable levels were also observed in VKC: BLT1 $(40.48 \pm 10) ; C 5 a R(44.90 \pm 20)$. Very few cells co-expressed both receptors while only rarely were any $\mathrm{BLT}^{+}$or $\mathrm{C} 5 \mathrm{aR} 1^{+}$cells detected in healthy mouse and human tissues, suggesting that these receptors are exclusively expressed during disease, potentially by infiltrating CD4 ${ }^{+} \mathrm{T}$ cells and $\mathrm{MC}$. 
(A) Day 7 of treatment

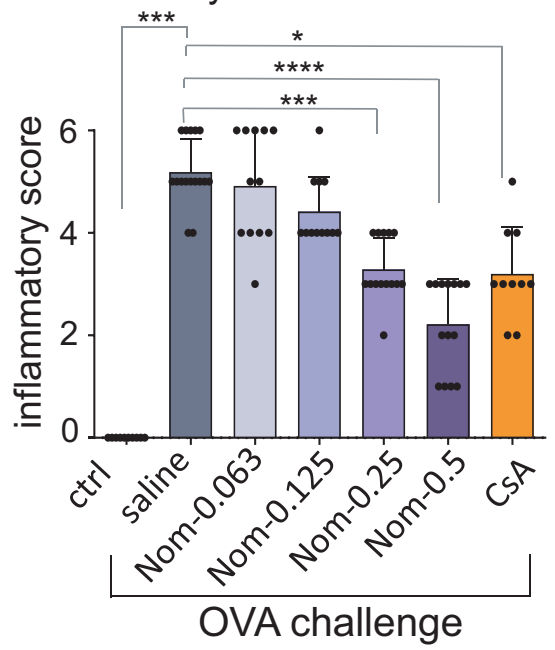

(D)
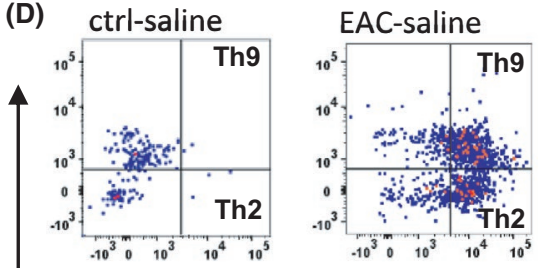

EAC-Nom-0.25

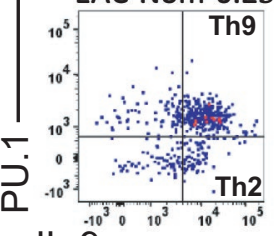

IL-9-
(B) Day 10 of treatment

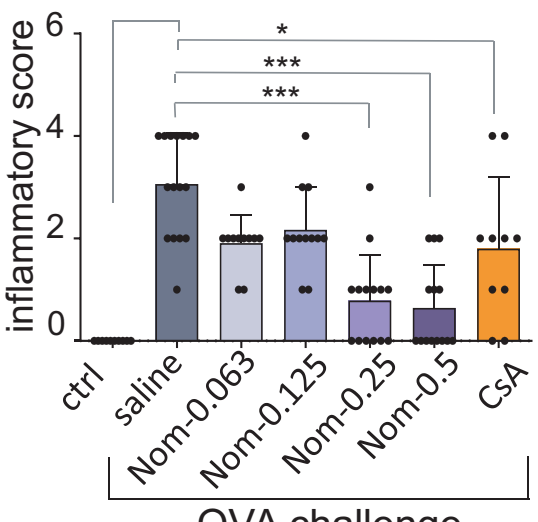

OVA challenge
(C) Day 10 of treatment

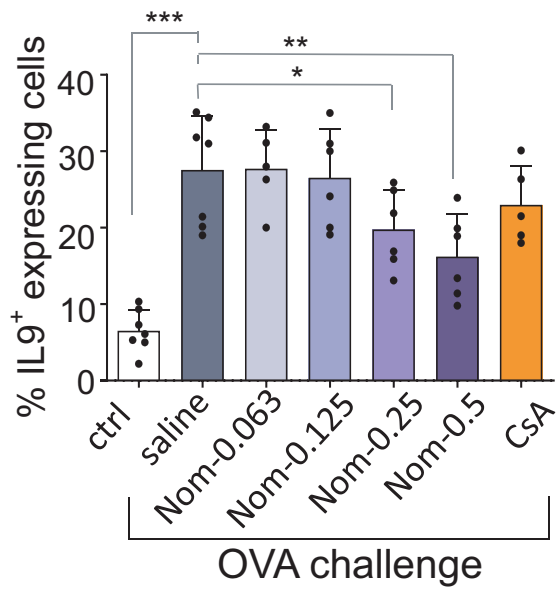

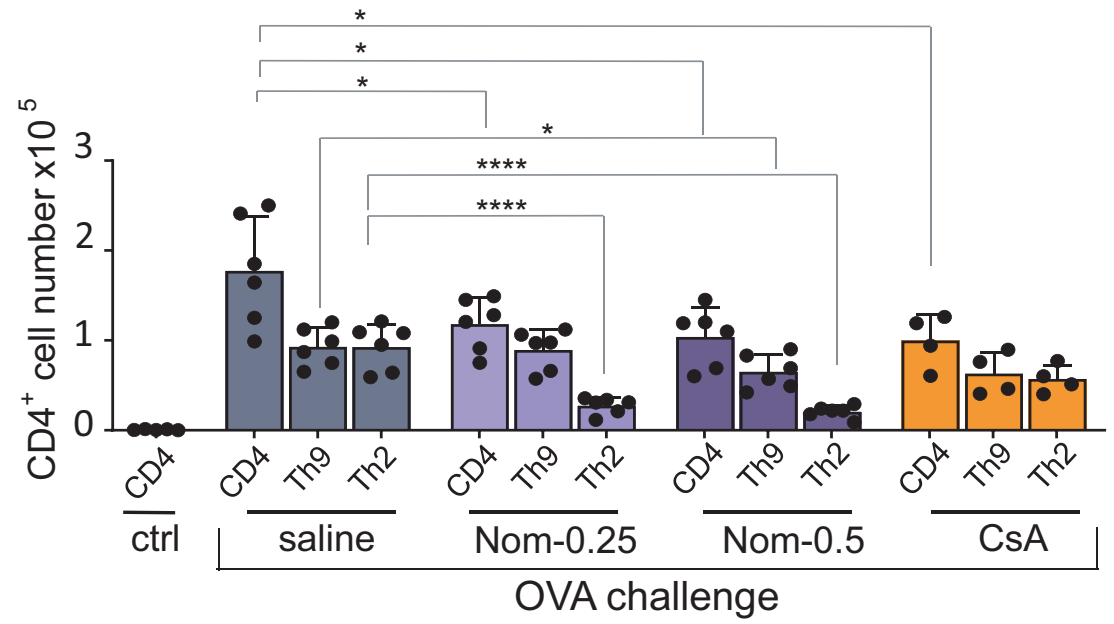

(F)

(E)

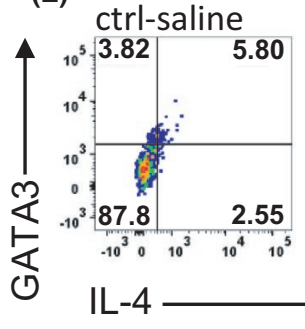

EAC-saline

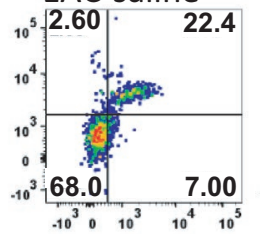

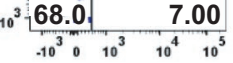

EAC-Nom-0.25 EAC-Nom-0.5

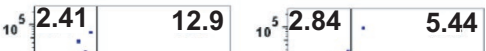

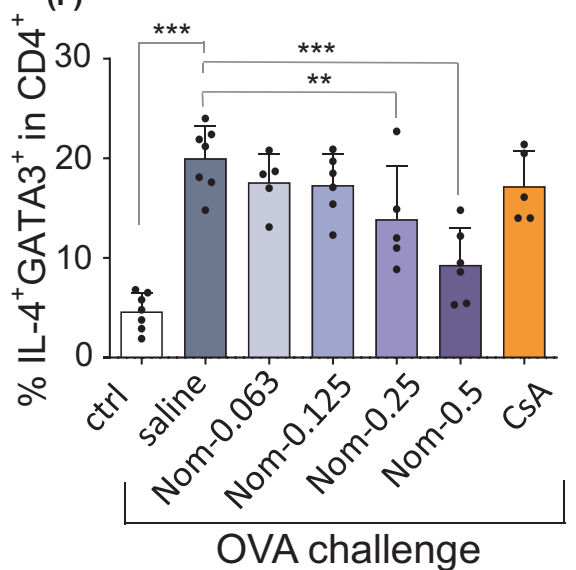

FIGURE 1 Topical nomacopan attenuated conjunctivitis in EAC and decreased IL-9 in CD4 ${ }^{+}$T cells. (A, B) Clinical scores of eyes were compared with saline-treated controls. (C-F) Conjunctival explant cells stained for T-cell markers. Flow cytometry plots represent staining for: (C) IL-9, (D) IL-9 and PU.1 (Th9 cells) and a summary graph of percentages for IL-9+PU.1 ${ }^{+}$(Th9) or IL-9+ PU.1- (Th2) cells. (E, F) Representative plots were gated for Th2 cells (IL- $4^{+}$GATA $3^{+} \mathrm{PU} .1^{-}$) in treatment groups and a summary graph. Four independent experiments, ANOVA and unpaired $t$-tests; mean \pm SD ( $n=6-8$ mice per group). ${ }^{*} p<.05,{ }^{* *} p<.01$. Nom $=$ nomacopan, CsA $=$ Cyclosporine $A$ 


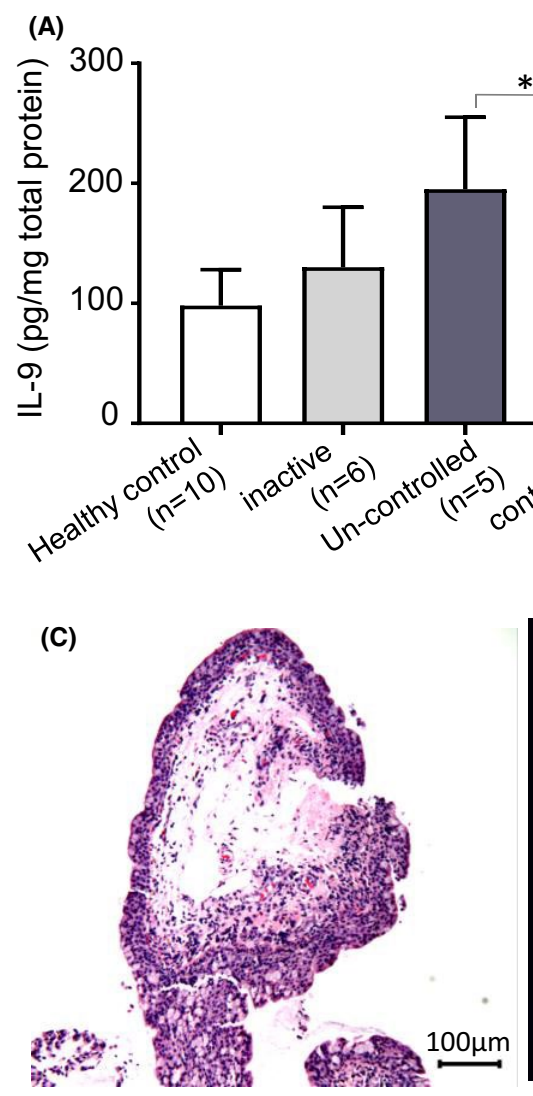

(D)

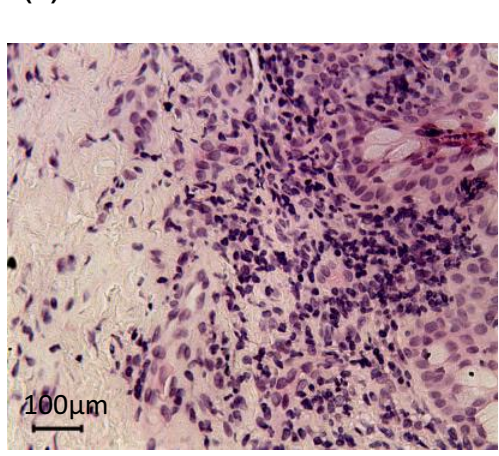

IL-9R

Typtase

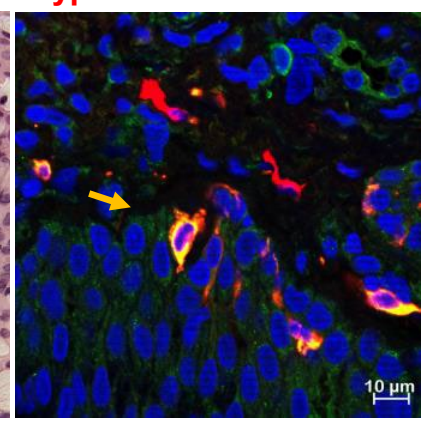

BLT1

(F)

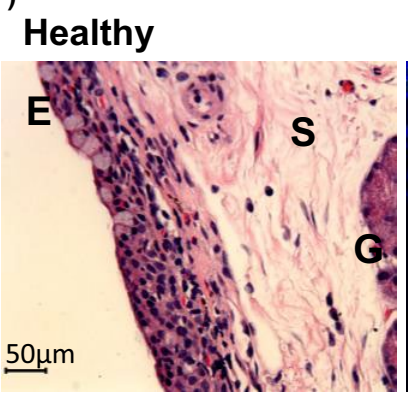

IL-9R

CD4

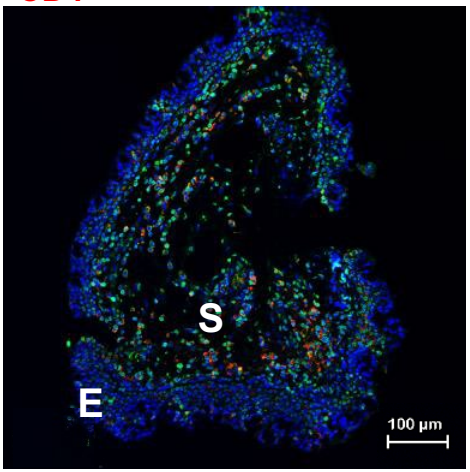

CD4=white

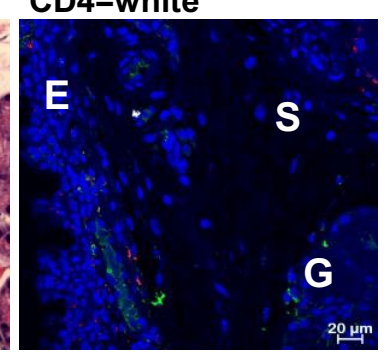

(G)
(B)

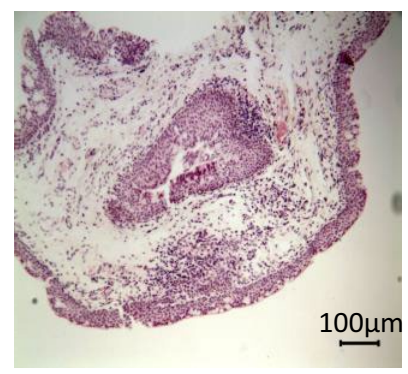

IL-9

CD4

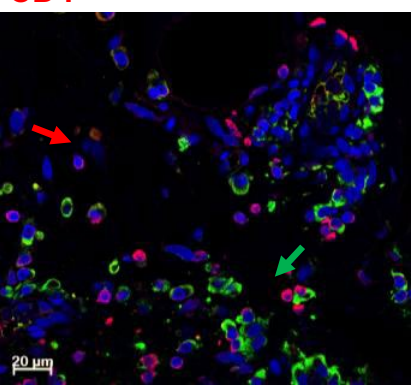

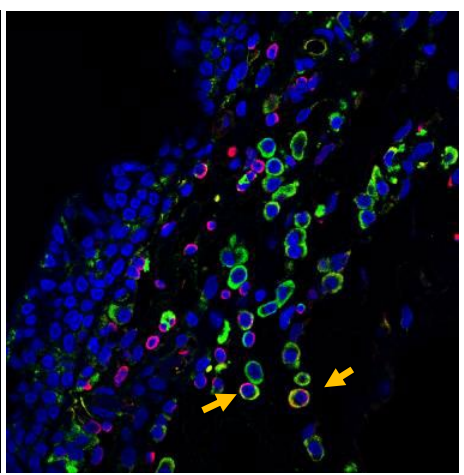

(E)

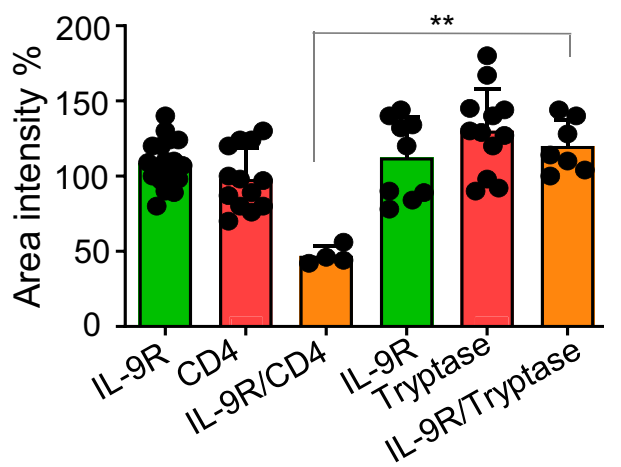

BLT1

C5aR

VKC

CD4=white
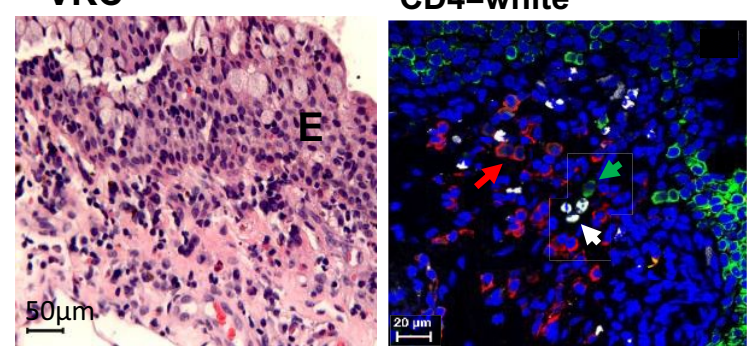

FIGURE 2 (A) IL-9 levels in tear fluids, demonstrating an increase in clinically uncontrolled (i.e. active) VKC as compared with those with clinically controlled disease; (B) Histology/immunofluorescence staining of VKC conjunctival sections for CD4 and IL-9; (C) CD4 and IL-9R; (D) Tryptase and IL-9R. (E) Scatterplots based on immunoreactivity and intensity for IL-9R, tryptase or IL-9R and CD4 as percentages of the immunostained areas. Representative summary graph of one of 7 biopsies. Values were added from 3 fields. (F, G) Representative staining for C5aR1, BLT1, CD4 on healthy, 1 of 3 (F) and VKC 1 of 7 (G) conjunctival tissues. Mean \pm SD, ANOVA, ${ }^{* *} p<.01,{ }^{* *} p<.001$. E $=$ epithelial layer; $\mathrm{S}=$ stroma: $\mathrm{G}=$ gland (Lacrimal) 
We conclude that IL-9 was up-regulated during VKC and EAC. Nomacopan significantly suppressed EAC severity, accompanied by a decrease in IL-9-producing Th2 cells, Th2 cells and to a lesser extent, Th9 cells, suggesting a key pro-inflammatory role for IL-9secreting $C D 4^{+} \mathrm{T}$ cells in allergic eye disease.

Our findings support nomacopan as a potential treatment for allergic eye disease due to its ability to down-regulate LTB4/C5 pathways in EAC.

\section{FUNDING INFORMATION}

This research was supported in part by a research collaborative grant from Akari Therapeutics (V.C.), the National Institute for Health Research (NIHR) Moorfields Biomedical Research Centre and NIHR Moorfields Clinical Research Facility, based at Moorfields Eye Hospital NHS Foundation Trust and UCL Institute of Ophthalmology. The views expressed are those of the authors and not necessarily those of the NHS, the NIHR or the Department of Health. M.N. and W.W-D. are employees of Akari Therapeutics.

\section{ACKNOWLEDGEMENT}

We would like to thank our support staff, in particular in the BSU, for their assistance.

\section{CONFLICT OF INTEREST}

No authors have any Conflicts of Interest with the exception of Drs. Nunn and Weston-Davies, who are both employees of Akari Therapeutics Inc.
${ }^{1}$ Ocular Immunology Group, UCL Institute of Ophthalmology, London, UK
${ }^{3}$ NIHR Moorfields Clinical Research Facility, Moorfields Eye Hospital, London, UK

${ }^{4}$ NIHR Biomedical Research Centre at Moorfields Eye Hospital NHS Foundation Trust \&, UCL Institute of Ophthalmology,

London, UK

${ }^{5}$ Akari Therapeutics plc, London, UK

${ }^{6}$ Ophthalmology Operative Complex Unit, University Campus Bio-Medico, Rome, Italy

\section{Correspondence}

Virginia L. Calder, UCL Institute of Ophthalmology, 11/43

Bath Street, London EC1V 9EL, UK.

Email: v.calder@ucl.ac.uk

\section{ORCID}

Malihe Eskandarpour (D) https://orcid.org/0000-0002-6380-5389

Virginia L. Calder (D) https://orcid.org/0000-0003-1422-2926

\section{REFERENCES}

1. Leonardi A, Lazzarini D, Motterle L, etal. Vernal keratoconjunctivitislike disease in adults. Am J Ophthalmol. 2013;155(5):796-803. doi:10.1016/j.ajo.2012.11.018

2. Reyes NJ, Mathew R, Saban DR. Induction and characterization of the allergic eye disease mouse model. Methods Mol Biol. 2018;1799:49-57. doi:10.1007/978-1-4939-7896-0_5

3. Roversi P, Ryffel B, Togbe D, et al. Bifunctional lipocalin ameliorates murine immune complex-induced acute lung injury. J Biol Chem. 2013;288(26):18789-18802. doi:10.1074/jbc.M112.420331

4. Sidik CD, Miyabe Y, Sezin T, Luster AD. The critical role of C5a as an initiator of neutrophil-mediated autoimmune inflammation of the joint and skin. Semin Immunol. 2018;37:21-29. doi:10.1016/j. smim.2018.03.002

5. Micosse C, von Meyenn L, Steck O, et al. Human "TH9" cells are a subpopulation of PPAR-gamma(+) TH2 cells. Sci Immunol. 2019;4(31):1-13. 10.1126/sciimmunol.aat5943

6. Chang HC, Sehra S, Goswami R, et al. The transcription factor PU.1 is required for the development of IL-9-producing T cells and allergic inflammation. Nat Immunol. 2010;11(6):527-534. https://doi. org/10.1038/ni.1867

\section{SUPPORTING INFORMATION}

Additional supporting information may be found in the online version of the article at the publisher's website.

${ }^{2}$ UOSD Research Laboratories, IRCCS-fondazione Bietti, 